\title{
Stereotactic Body Radiosurgery Using Volumetric Arc Therapy as an Alternative to High Dose Rate Brachytherapy Boost in Gynecological Cancer
}

\author{
Beatriz E. Amendola ${ }^{1}$, Naipy Perez ${ }^{2}$, Marco a. Amendola ${ }^{2}$, Xiaodong Wu ${ }^{1}$ \\ 1. Innovative Cancer Institute 2. Innovative Cancer Institute, 6141 Sunset Drive, South Miami, FL 33143
}

$\square$ Corresponding author: Beatriz E. Amendola, dramendola@gmail.com

Disclosures can be found in Additional Information at the end of the article

\section{Abstract}

Background: The purpose of this study was to determine if stereotactic body radiosurgery (SBRT) using volumetric modulated arc therapy (VMAT) can be used to deliver a boost to the vaginal cuff in patients with gynecological cancer as an alternative to high dose rate (HDR) brachytherapy.

Methods: Between January and July 2009, five patients with gynecological cancer were treated with postoperative radiation therapy to the whole pelvis with doses ranging from $41.4 \mathrm{~Gy}$ to 45 Gy. This was followed with a boost to the vaginal cuff with SBRT using VMAT. The total dose delivered in the boost ranged from $9 \mathrm{~Gy}$ to $21 \mathrm{~Gy}$ given in three fractions. Plan comparisons between SBRT with VMAT and HDR were performed for each patient.

Results: After three years follow up (range 36 - 43 months), four of five patients (80\%) are alive and well, except for one who expired of intercurrent disease after two years. Mild acute toxicity was seen in two out of five patients (40\%). No chronic toxicity has been encountered. The dose coverage to the vaginal cuff using either HDR or VMAT was similar; however, the VMAT plan provided superior dose uniformity throughout the target, as well as better sparing of the bladder and the rectum at the mid to high dose levels.

Conclusions: In this case review, target coverage, tolerance, and toxicity using SBRT with VMAT as a boost to the vaginal cuff were comparable to HDR brachytherapy. This technique could be considered as a potential alternative to HDR brachytherapy.

Review began 11/12/2012 Published 11/20/2012

C) Copyright 2012

Amendola et al. This is an open access article distributed under the terms of the Creative Commons Attribution License CC-BY 3.0., which permits unrestricted use, distribution, and reproduction in any medium, provided the original author and source are credited.
Categories: Obstetrics/Gynecology, Radiation Oncology

Keywords: high dose rate brachytherapy, stereotactic body radiosurgery, stereotactic body radiation therapy, volumetric modulated arc therapy (vmat), Stereotactic Radiosurgery, brachytherapy, gynecology oncology, cyberknife $\subset$, gynecological cancers

\section{Introduction}

Cancer of the endometrium is the most common gynecologic cancer in the United States [1]. The standard management for endometrial cancer is a total abdominal hysterectomy and bilateral salpingo-oophorectomy (TAH/BSO) with or without pelvic lymph node sampling. External beam radiation therapy (EBRT) and/or brachytherapy are integral components in the postoperative adjuvant therapy of selected patients [2]. Brachytherapy is being increasingly

\section{How to cite this article}

Amendola B E, Perez N, Amendola M a., et al. (November 20, 2012) Stereotactic Body Radiosurgery Using 


\section{Cureus}

delivered by high dose rate (HDR) techniques to boost the vaginal cuff in high risk patients [34]. There are cases where brachytherapy is either not available or not possible, such as in claustrophobic patients who cannot tolerate being treated in an enclosed vault. In this report, we explore the feasibility of treating gynecologic cancer with stereotactic body radiosurgery (SBRT) with volumetric arc therapy (VMAT) as an alternative option to intracavitary HDR Brachytherapy for vaginal cuff boost.

\section{Case Presentation}

\section{Methods}

Between January and July 2009, after informed consent was obtained, five patients with gynecological cancer: four with endometrial and one with cervical cancer with ages ranging from 37 to 77 years (mean: 62, median: 69) were treated with postoperative radiation therapy to the whole pelvis with doses ranging from $41.4 \mathrm{~Gy}$ to $45 \mathrm{~Gy}$. Patient characteristics are described in Table 1. All patients underwent initial surgical management consisting of $\mathrm{TAH} / \mathrm{BSO}$ with pelvic plus/minus para-aortic lymph node sampling. These five patients were treated with postoperative pelvic radiotherapy because of their high risk factors. Vaginal boost was indicated as part of adjuvant therapy to reduce the risk of recurrence. However, these patients were not candidates for HDR because of claustrophobia. In order to optimize treatment management, we elected to treat with stereotactic body radiosurgery (SBRT) with volumetric modulated arc therapy (VMAT).

\begin{tabular}{|c|c|c|c|c|c|c|c|c|}
\hline & Age & Diagnosis & Pathology & Site & Stage & Treatment & F/U (months) & Status \\
\hline CASE 1 & 70 & Uterine CA & Clear Cell Carcinoma & Uterus & IIIC & S, CTX, RT & 43 & NED \\
\hline CASE 2 & 69 & Uterine CA & Carcinosarcoma & Uterus & IA & S, CTX, RT & 39 & NED \\
\hline CASE 3 & 77 & Uterine CA & Adenocarcinoma & Uterus & IC & S, RT & 37 & Deceased \\
\hline CASE 4 & 37 & Cervical CA & Squamous Cell Carcinoma & Cervix & IB & S, CTX, RT & 37 & NED \\
\hline CASE 5 & 59 & Uterine CA & Adenocarcinoma & Uterus & IB & S, RT & 37 & NED \\
\hline
\end{tabular}

\section{TABLE 1: Patient Characteristics}

S: Surgery CTX: Chemotherapy RT: Radiation Therapy

CT simulation was performed in all patients and to better identify and delineate the clinical target volume, an HDR vaginal cylinder was used for immobilization and localization purposes. Image acquisitions with $3 \mathrm{~mm}$ thickness CT slices over the region of interest were performed. The planning treatment volume (PTV) was outlined following the guidelines for the contouring of structures for HDR Brachytherapy treatments. This was accomplished by using a $5 \mathrm{~mm}$ margin around the upper $5 \mathrm{~cm}$ of the cylinder and $1 \mathrm{~cm}$ superiorly. The HDR brachytherapy cylinder was used for CT planning, as well as for treatment in all patients. Cone beam CT (CBCT) was performed as the image-guided radiation therapy (IGRT) method prior to every treatment fraction to confirm the correct setup. The total dose delivered for the boost ranged from $9 \mathrm{~Gy}$ to $21 \mathrm{~Gy}$, given in three fractions. The prescription dose was individualized depending on the specific characteristics of the patient. The total biological effective dose delivered ranged from $54 \mathrm{~Gy}$ to $74 \mathrm{~Gy}$ with a mean of $64 \mathrm{~Gy}$ using an $\alpha / \beta$ ratio of $10 \mathrm{~Gy}$, including the dose delivered with EBRT, in addition to the SBRT boost. 


\section{Cureus}

Planning was performed with the Eclipse Planning System ${ }^{\mathrm{TM}}$ using two or three coplanar arcs. A Varian TrilogyTM linear accelerator was used for the VMAT (RapidArcTM) treatment delivery.

For the comparison between SBRT with VMAT and HDR brachytherapy boost, treatment plans were created using the same CT images in all patients. Figures 1,2 represent the isodose distributions of both HDR and VMAT plans at the mid-level of the cylinder in one of our patients.

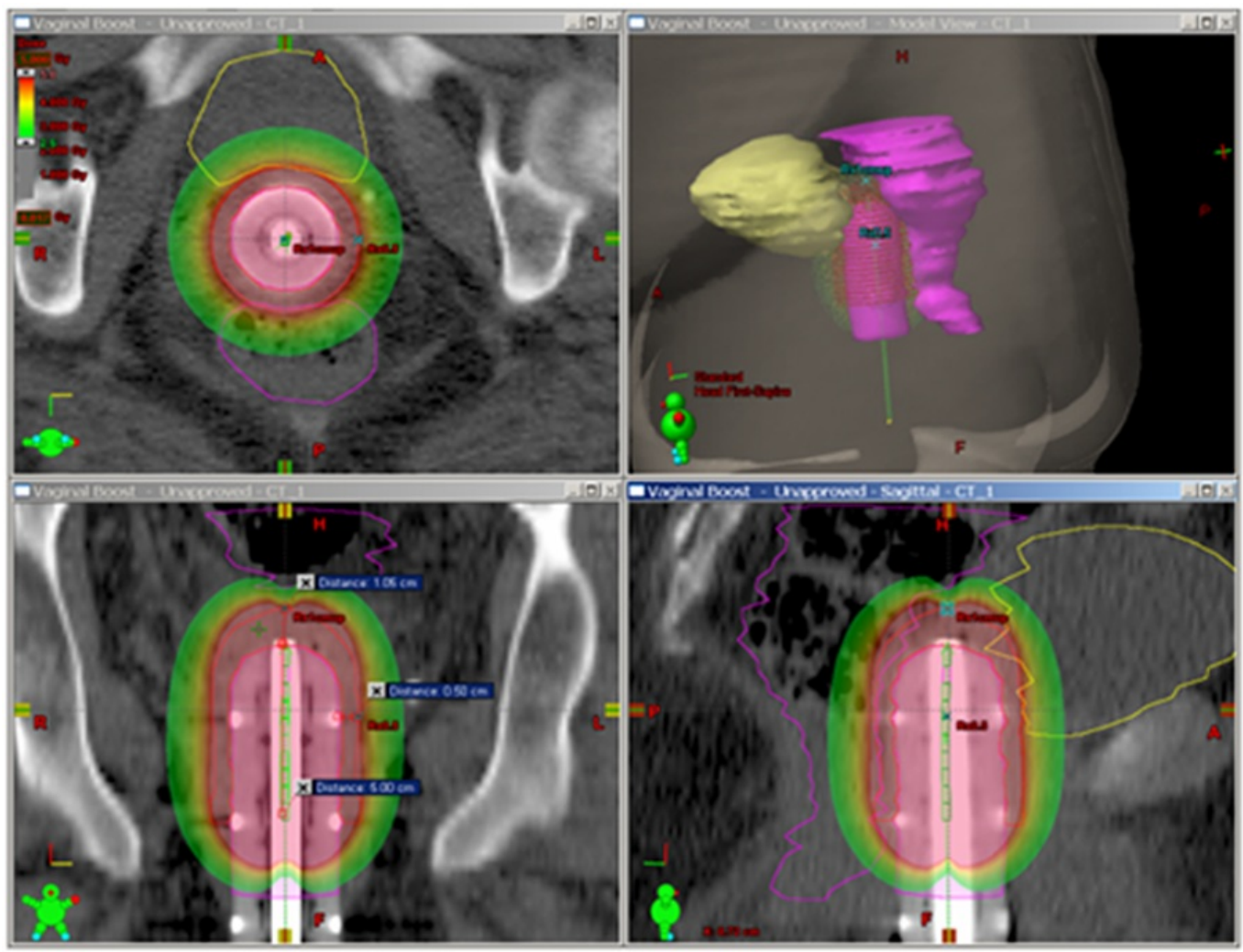

FIGURE 1: Representative isodose distribution in colorwash

Representative isodose distribution in colorwash for an HDR Brachytherapy plan with a vaginal cylinder for a boost to the vaginal cuff after external beam radiation therapy. 


\section{Cureus}

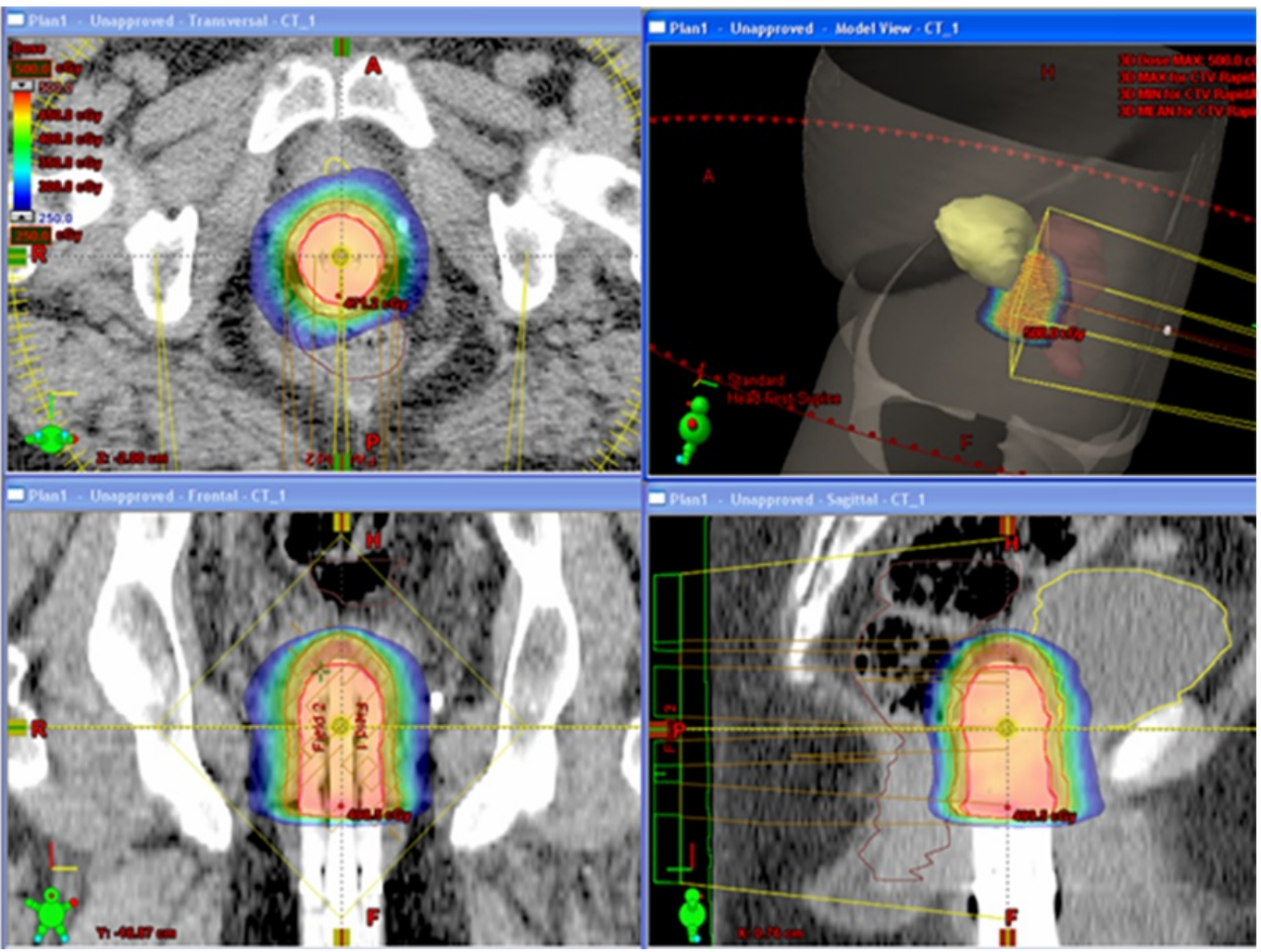

\section{FIGURE 2: Isodose distribution in colorwash}

Isodose distribution in colorwash for an SBRT plan using volumetric modulated arc therapy to treat the vaginal cuff after external beam radiation therapy to the pelvis. An HDR vaginal cylinder is used for delineation, immobilization and visualization of the CTV.

The comparisons were based on the dose volume histograms (DVH) to assess the dose distribution in the PTV as well as the dose to adjacent organs at risk (OAR), i.e. bladder and rectum. Figure 3 shows a comparison between the DVH obtained from a HDR and RapidArcTM plans, respectively, in a representative patient.
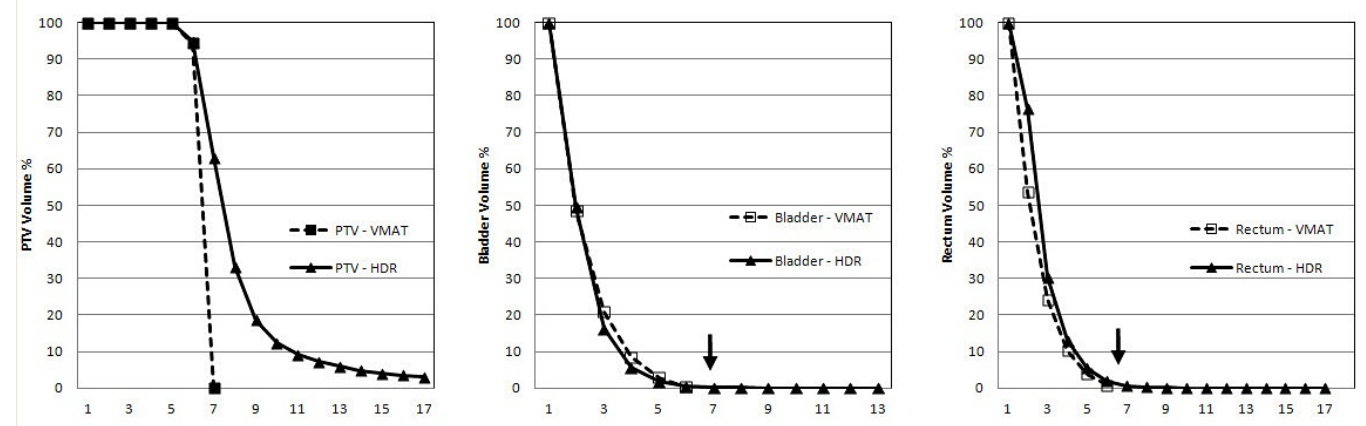

FIGURE 3: DVH comparison between SBRT with VMAT vs. HDR

\section{Brachytherapy plans}

DVH comparison between SBRT with VMAT vs. HDR Brachytherapy plans. Dashed and solid lines represent the doses in the VMAT and HDR plans. Both plans provide comparable coverage of the target volume. However, as shown in Figure $3 A$ the VMAT plan provides superior dose uniformity throughout the target. Figure $3 \mathrm{~B}$ and Figure $3 \mathrm{C}$ show comparable 


\section{Results}

After a minimum of three years follow up (range 36 - 43 months, mean: 38 months), four of five (80\%) are alive and well, except for one patient who expired of intercurrent disease after two years. None of the patients developed severe acute toxicity. Mild acute toxicity was seen in two out of five patients (40\%) consisting of vaginal mucosal discharge and transient urinary irritation. No chronic toxicity has been encountered.

Comparing HDR with SBRT using VMAT, both techniques offer similar dose coverage in the PTV with more than $95 \%$ of the volume receiving $100 \%$ of the prescription dose. However, the volume of tissue in the PTV receiving doses higher than the prescription dose is considerably greater for HDR than VMAT. The VMAT plan provides superior dose uniformity throughout the target and a sharper drop off of the dose (Figure $3 \mathrm{~A}$ ). The dose distribution in the bladder and rectum are similar for doses lower than the prescription dose (Figure 3B, Figure 3C). However, the maximum point doses for the OAR are considerably higher using HDR due to the dose inhomogeneity. A comparison of the DVHs for these plans is shown in Figure 3. The HDR plans had a higher degree of dose non-uniformity compared to the VMAT plan. Beam on delivery time ranged from $2.5 \mathrm{~min}$ to $4.4 \mathrm{~min}$ with a mean value of 3.4 minutes.

\section{Discussion}

Both plans provided similar coverage of the target volume, however, the VMAT plan achieved superior dose uniformity throughout the target, as well as better sparing of the bladder and the rectum at the mid to high dose levels. Although the treatment time using both techniques is comparable, the setup time of the patient prior to treatment delivery with EBRT boost using SBRT with VMAT is considerably longer than the usual setup time with HDR. Besides verifying that the patient is in the correct position, it is also essential to guarantee that the location of the cylinder in relation to the patient is precise. This is achieved by using IGRT with CBCT. The feasibility of SBRT with VMAT as an alternative to HDR boost in women with early gynecological cancer is being suggested [3-4]. Early data from the group of Molla, et al. demonstrates that use of radiosurgery to deliver a final boost to the areas at higher risk for relapse in endometrial or cervical cancer is feasible, well-tolerated and may be considered as an acceptable alternative to HDR brachytherapy [3]. Mayr and Deodato, et al. described the new paradigm of SBRT in the management of gynecological cancer mostly for recurrences with high success in local control [5-6]. The accuracy of the treatment delivery has been further improved with the use of daily image-guided radiation therapy.

\section{Conclusions}

SBRT with VMAT to the vaginal cuff was comparable to HDR brachytherapy. The VMAT plans had a more homogeneous distribution of the dose in the PTV. Homogeneity index (HI) was 2.66 for HDR and 1.16 for VMAT. After three years of follow-up, we feel confident that this alternative technique could be offered to patients with gynecological tumors where brachytherapy is not possible due to inability of the patient to undergo treatment in some centers where the brachytherapy vault is very small or when brachytherapy is not available. SBRT with VMAT delivers highly focused radiation, is exceedingly precise, and can be given in a shorter time than conventional radiation. IGRT adds to the safety and precision of the procedure. Our case review demonstrates that SBRT with VMAT can be used successfully to deliver a boost after pelvic irradiation in patients with gynecological cancer. Further investigation of this alternative technique is warranted. 


\section{Additional Information \\ Disclosures}

Human subjects: Consent was obtained by all participants in this study. Conflicts of interest: In compliance with the ICMJE uniform disclosure form, all authors declare the following:

Payment/services info: All authors have declared that no financial support was received from any organization for the submitted work. Financial relationships: All authors have declared that they have no financial relationships at present or within the previous three years with any organizations that might have an interest in the submitted work. Other relationships: All authors have declared that there are no other relationships or activities that could appear to have influenced the submitted work.

\section{References}

1. Cancer Facts \& Figures. 2012,

2. Aydogan B, Mundt AJ, Smith BD, et al.: A dosimetric analysis of intensity-modulated radiation therapy (IMRT) as an alternative to adjuvant high-dose-rate (HDR) brachytherapy in early endometrial cancer patients. Int J Radiation Oncology Biol Phys. 2006, 65:266-273. 10.1016/j.ijrobp.2005.12.049

3. Mollà M, Escude L, Nouet $P$, et al.: Fractionated stereotactic radiotherapy boost for gynecologic tumors: An alternative to brachytherapy?. Int J Radiation Oncology Biol Phys. 2005, 62:118-124. 10.1016/j.ijrobp.2004.09.028

4. Higginson DS, Morris DE, Jones EL, Clarke-Pearson D, Varia MA: Stereotactic body radiotherapy (SBRT): Technological innovation and application in gynecologic oncology. Gynecol Oncol. 2011, 120:404-412. 10.1016/j.ygyno.2010.11.042

5. Mayr NA, Huang Z, Sohn JW, et al.: Emerging application of stereotactic body radiation therapy for gynecologic malignancies. Expert Rev Anticancer Ther. 2011, 11:1069-1075. 10.1586/era.11.81

6. Deodato F, Macchia G, Grimaldi L, et al.: Stereotactic radiotherapy in recurrent gynecological cancer: A case series. Oncol Rep. 2009, 22:415-419. 